\title{
INTEGRAL/SPI and Fermi/GBM Observations of the 2012 March 7th Solar Flares
}

\author{
Xiao-Ling Zhang* \\ Max-Planck Institute for Extraterrestrial Physics \\ Giessenbachstr. 1, D-85748, Garching, Germany \\ E-mail: zhangxdmpe.mpq.de

\section{David Gruber} \\ MPE, Garching, Germany \\ E-mail: dqruberdmpe.mpg.de

\section{Jürgen Kiener} \\ CSNSM, Orsay, France \\ E-mail: Gurgen.kienerdcsnsm.in2p3.fr
}

\section{Andreas von Kienlin}

MPE, Garching, Germany

E-mail: azkdmpe.mpg.de

\begin{abstract}
We present preliminary results of INTEGRAL/SPI and Fermi/GBM observations of the X5.4 and X1.3 solar flares on 2012 March 7. The GOES X5 flare commenced at 00:04 UT and was followed by the X1 flare an hour later. The entirety of both flares was detected by INTEGRAL/SPI, whereas the Fermi/GBM observed the falling phase of the X5 and entirety of the X1 flare. The $2.2 \mathrm{MeV}$ neutron capture line is very prominent in both flares as observed by SPI. We performed Monte Carlo simulations to obtain the response of the SPI matrix in such a non-standard configuration, in order to calculate the flux of the $2.2 \mathrm{MeV}$ line measured with SPI. Our preliminary study shows that the $2.2 \mathrm{MeV}$ flux obtained with SPI for the $\mathrm{X} 1$ flare is around $30 \%$ smaller than that obtained with Fermi/GBM. There are variety of reasons for this difference including uncertainty in the SPI response and background subtraction in the GBM.
\end{abstract}

"An INTEGRAL view of the high-energy sky (the first 10 years)" 9th INTEGRAL Workshop and celebration of the 10th anniversary of the launch,

October 15-19, 2012

Bibliotheque Nationale de France, Paris, France

\footnotetext{
* Speaker.
} 


\section{Introduction}

Gamma ray lines contain important information on particle acceleration in the solar atmosphere. Line intensities and their temporal evolution and line shape studies give information on properties of the solar atmosphere and the accelerated particle composition and their momenta and angular distributions [四]. Line shape studies which require high energy resolution spectroscopy, have been applied to several solar flares since the launch of the Reuven Ramaty High Energy Solar

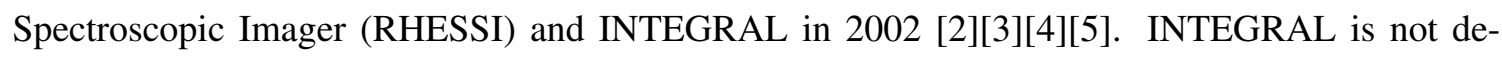
signed for studying solar physics, but with its Germanium camera's high energy resolution and the 3 days uninterrupted observation, it makes a unique instrument to study gamma-ray lines in strong solar flares.

A strong solar flare classified by the Geostationary Operational Environmental Satellites (GOES) as $\mathrm{X} 5.4^{1}$, commenced on 2012 March 7th, at 00:04 UT. A second, weaker flare (X1.3) occurred about an hour later. INTEGRAL detected the flare at 00:15 UT, and observed the two flares in their entirety, whereas Fermi/GBM missed the first flare because it was behind the Earth. RHESSI was also behind the Earth during the first flare.

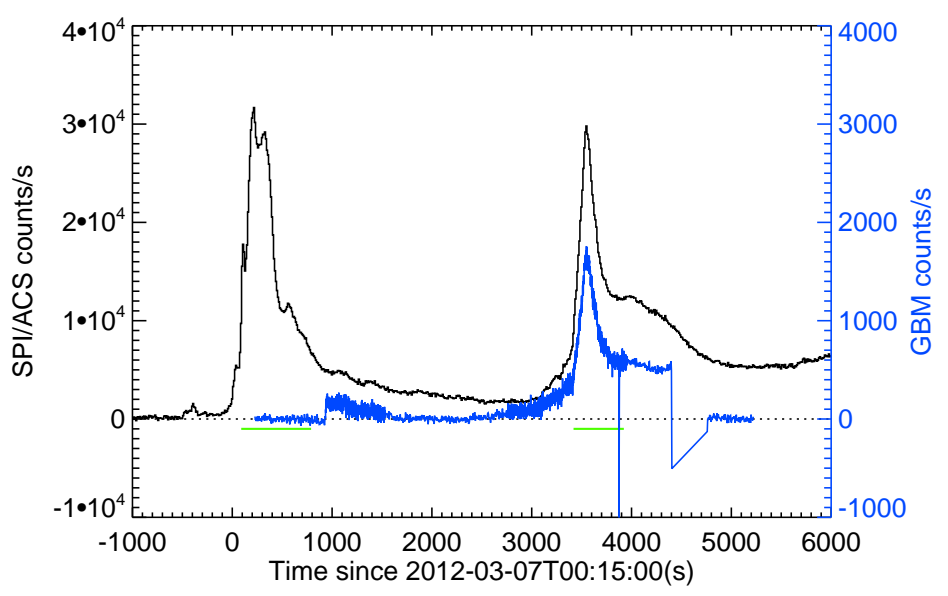

Figure 1: The background subtracted SPI/ACS (black) and the Fermi/GBM light curve (blue)

Figure $\square$ shows the background subtracted SPI/ACS (above $100 \mathrm{keV}$ ) and GBM (250 keV to $40 \mathrm{MeV}$ ) light curves of the two flares. The green bars mark the time range in which the flare fluxes were calculated (see Sections $[2.3$ and $\mathbf{A}$ ).

\section{Observation with INTEGRAL/SPI Ge detectors}

During the solar flares, INTEGRAL was pointing at the inner Galactic disk and the Sun was in the XZ plane, about 83 degrees from the SPI axis. Although the Sun was very far from the SPI field of view, some of the radiation from both flares penetrated the thick BGO ACS and was detected by the Germanium detector matrix, thus allowing the study of gamma ray lines from the Sun with SPI's very high energy resolution.

\subsection{Ge detector counts}

Figure $\square$ shows the raw count rates of Ge detectors in a few energy ranges. The bursts are significant in the continuum and at the $2.2 \mathrm{MeV}$ line produced by neutron being captured by hydrogen and forming Deuterium $\left({ }^{1} \mathrm{H}+\mathrm{n} \rightarrow \mathrm{D}+\gamma\right)$.

\footnotetext{
${ }^{1} \mathrm{X}$-ray flux in the 1 to $8 \AA$ band $=5.4 \times 10^{-4} \mathrm{~W} / \mathrm{m}^{2}$, see http://www.swpc.noaa.gov/info/glossary.html
} 
- Panel 1: 20-200 keV continuum

- Panel 2: neutron capture line at 2223.4 $\mathrm{keV}$. The long tail is probably due to the 100 second exponential due to neutron slow down and capture.

- Panel 3: 4.4 MeV, ${ }^{12} \mathrm{C}^{*} \beta$-decay line

- Panel 4: 6.1 MeV, ${ }^{16} \mathrm{O} *$ nuclear deexcitation lines

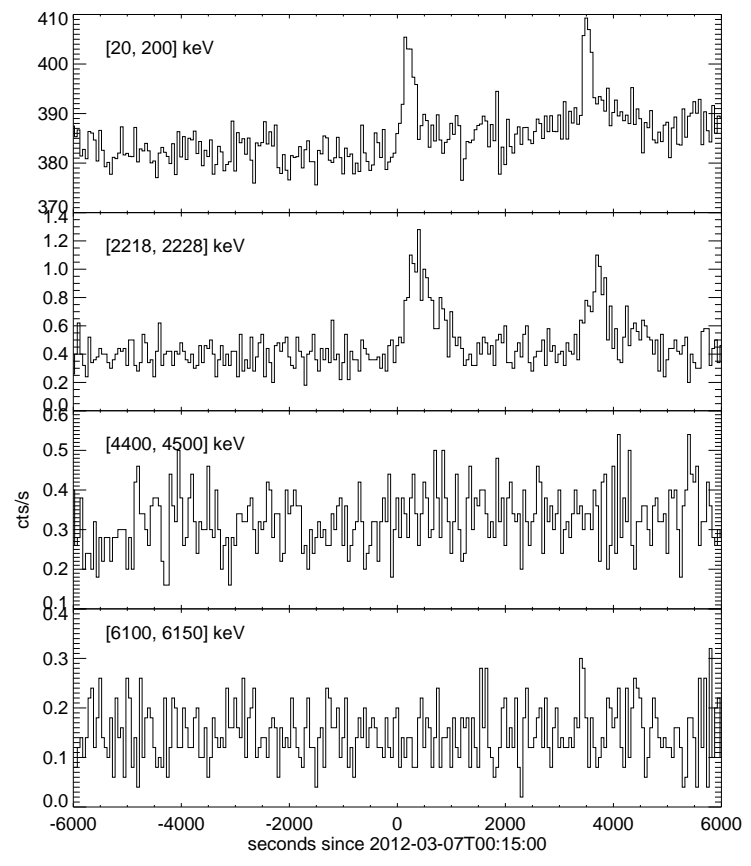

Figure 2: Count rate of the Ge-detectors in 50s bin.

\subsection{The 2.2 MeV neutron capture line spectrum}

As shown in Figure 凹, the neutron capture line at $2.2 \mathrm{MeV}$ was very strong in both flares, whereas the nuclear de-excitation lines were not immediately visible without employing a more detailed analysis, which is to be carried out. In order to estimate the strength of the $2.2 \mathrm{MeV}$ neutron capture line, we selected time intervals of interest and obtain the count spectra and deadtime information with the official INTEGRAL Off-line Scientific Analysis ${ }^{2}$ (OSA) tools. As background we chose 3 pointings, with a total elapsed time 5577 seconds, 9 minutes before the flares. Figure 3$]$ shows the spectra of the 3 pointings used as background (top), the spectra at the two flares after background subtraction (middle, bottom). A Gaussian was fitted to the $2.2 \mathrm{MeV}$ line while a polynomial was used for the continuum (blue lines). The fitted parameters of the Gaussian component would be used to estimate the flux/fluence of the $2.2 \mathrm{MeV}$ line.

\footnotetext{
${ }^{2} \mathrm{http}: / /$ www.isdc.unige.ch/integral/analysis
}

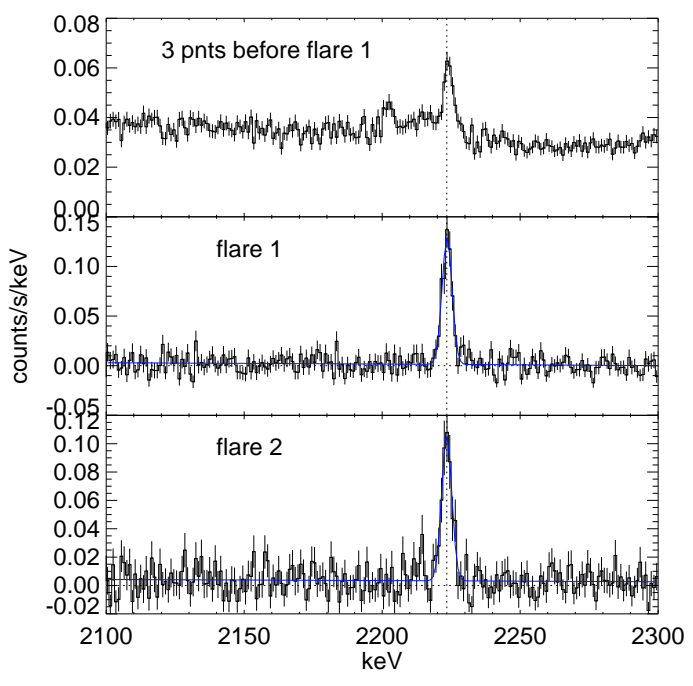

Figure 3: SPI spectra of the two flares around the 2.2 MeV neutron capture line 


\subsection{SPI response in this uncommon configuration}

The direction of the Sun with respect to the satellite during the two flares is shown in Figure 8 ; around 83 degrees from the SPI pointing direction, thus the incident photons from the Sun must go through the satellite structure, IBIS and the SPI anti-coincidence shield. To obtain the instrument response matrix in this uncommon configuration, we performed Monte-Carlo simulations[[5] with $4 \times 10^{6}$ incident photons, taking into account the loss of $4 \mathrm{Ge}$ detectors. The effective area at $2.2235 \mathrm{MeV}$ is $5.02 \pm 0.05 \mathrm{~cm}^{2}$ for the first flare

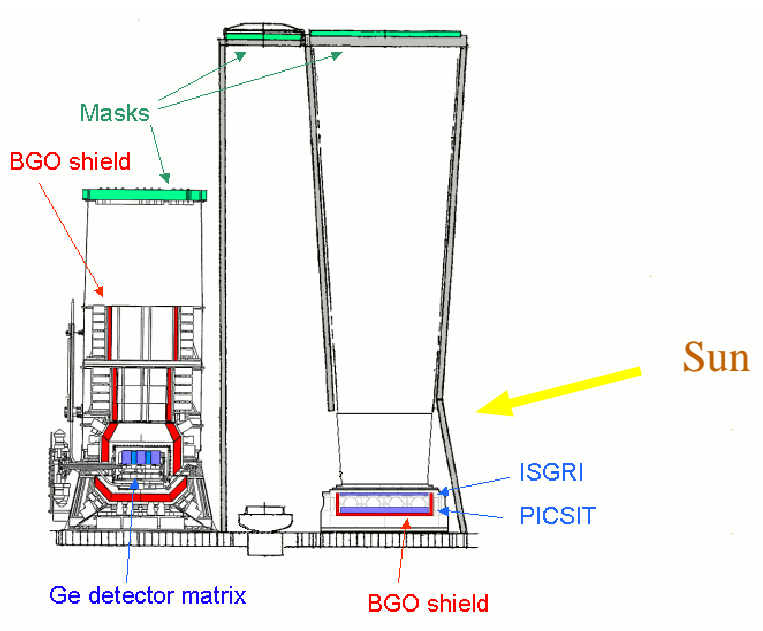

Figure 4: INTEGRAL model , and $5.03 \pm 0.05 \mathrm{~cm}^{2}$ for the second flare. Obviously there is no significant difference, which is expected for nearly identical observation angles.

The flux of the neutron capture line at the two flares in the selected time ranges (marked by the two green bars in Figure $\mathbb{W}$ ) is estimated to be: $0.116 \mathrm{ph} / \mathrm{s} / \mathrm{cm}^{2}$ and $0.094 \mathrm{ph} / \mathrm{s} / \mathrm{cm}^{2}$, respectively. The first flare lasted more than 1200 seconds at around $2.2 \mathrm{MeV}$, and the total fluence of the neutron capture line is $85 \pm 8 \mathrm{ph} / \mathrm{cm}^{2}$. For the second flare the neutron capture line fluence is $65 \pm 7 \mathrm{ph} / \mathrm{cm}^{2}$.

\section{Fermi/GBM Observations}

GBM [ [ ] triggered and located the very bright and energetic X5.4 class solar flare on 2012

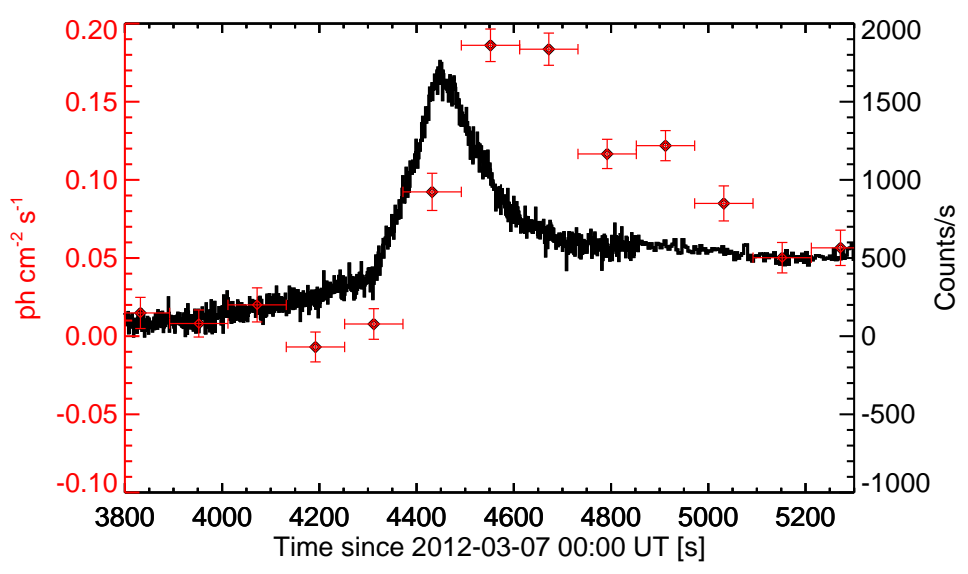

Figure 5: GBM BGO (250 keV to $40 \mathrm{MeV}$ ) light curve (black, same as in Figure 1) and $2.2 \mathrm{MeV}$ neutron capture line flux in 120 second time bin(red, preliminary, David Gruber in preparation) 


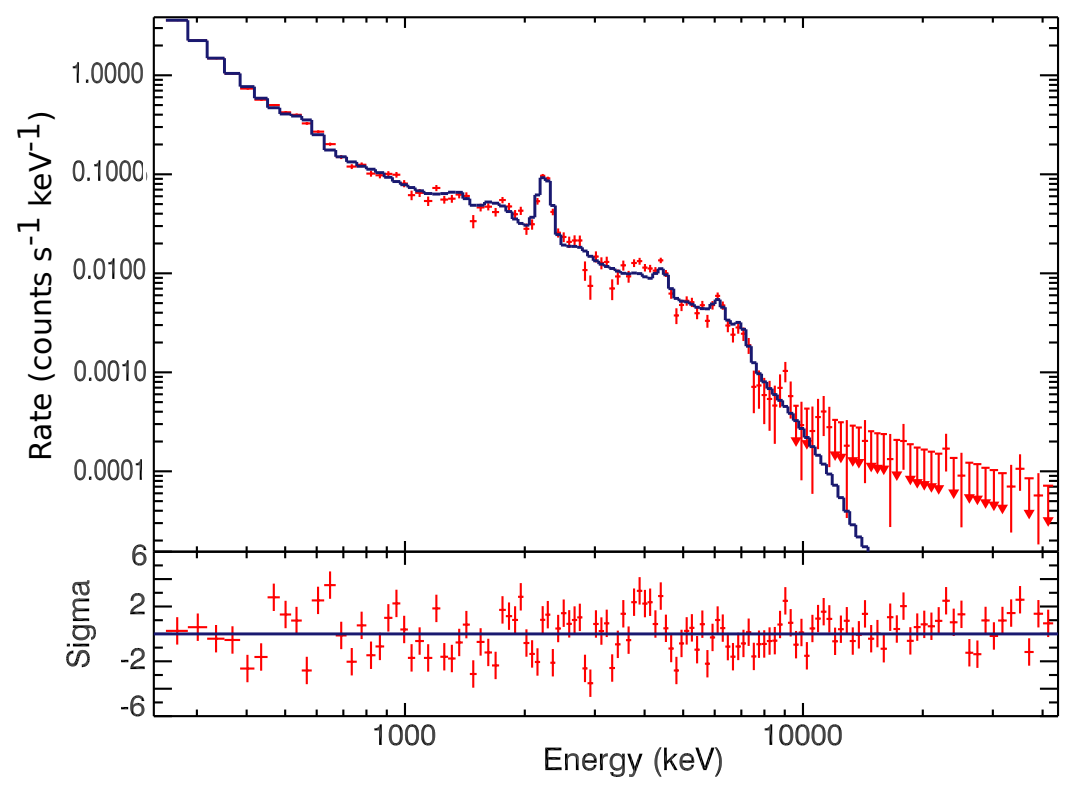

Figure 6: GBM count spectrum with fitted model and residual (preliminary)

March 7th, at 00:30:32.13 $\left(t_{0}\right)$ and triggered another 3 times on a subsequent X1 class solar flare at 01:00:57.53 UT, 01:10:58.20 UT and 02:06:09.93 UT. Most of the first solar flare was occulted by Earth so that only the last part of the prompt emission epoch is visible in GBM. The X1 solar flare, which followed only 30 min later, was visible until GBM entered the SAA during which the detectors were turned off. After exiting the SAA the solar flare was occulted by the Earth and only when it reappeared $1 \mathrm{~h}$ after the second trigger, GBM was able to observe the last minutes of this solar event.

One way to estimate the background count rates for long events in the NaI and BGO detectors has been proposed by Fitzpatrick et al. [8]], labeled "Orbital Subtraction Method". In short, they use the background rate of adjacent days, when the satellite has the same geographical footprint. The major limitation of this method is that there are still some systematic differences in the background rates when comparing -30 to +30 orbits after the activation following an SAA passage of Fermi.

Here, the "Solar Background Employing Relative Rates" $\left(\mathrm{SOBER}^{2}\right)$ method is used [6]. The working principle is that the specific detector configuration of GBM can be useful in determining the background rate of the sun-facing BGO 0 detector using the observed count rate of the Sun-occulted BGO 1 as a priori information. The major caveat of this method is that it implicitly assumes that no signal from the Sun is observed in the occulted BGO 1, which is a problem especially at lower energies where some photons will be collected in the BGO 1 due to scattering in the spacecraft.

The time integrated photon flux and fluence of the $2.2 \mathrm{MeV}$ neutron capture line in the selected time ranges (see Figure $\mathrm{W}$ ) are $0.132 \pm 0.005 \mathrm{ph} \mathrm{cm}^{-2} \mathrm{~s}^{-1}$ and $67 \pm 3 \mathrm{ph} \mathrm{cm}^{-2}$, respectively.

The evolution of the neutron capture line over the course of the X1 flare is presented in Figure [ and a count spectrum of the time integrated fit is presented in Figure 6. 


\section{Comparison of SPI and GBM results}

Table 1: SPI and GBM results of the $2.2 \mathrm{MeV}$ line

\begin{tabular}{lll}
\hline \hline & X5 flare & X1 flare \\
\hline \hline duration* $(\mathrm{s})$ & 700 & 504 \\
GBM flux $\left(\mathrm{ph} / \mathrm{s} / \mathrm{cm}^{2}\right)$ & & $0.132 \pm 0.005$ \\
GBM fluence $\left(\mathrm{ph} / \mathrm{cm}^{2}\right)$ & & $67 \pm 3$ \\
SPI flux $\left(\mathrm{ph} / \mathrm{s} / \mathrm{cm}^{2}\right)$ & $0.115 \pm 0.008$ & $0.094 \pm 0.009$ \\
SPI fluence $\left(\mathrm{ph} / \mathrm{cm}^{2}\right)$ & $80 \pm 6$ & $47 \pm 5$ \\
\hline SPI fluence total $* *\left(\mathrm{ph} / \mathrm{cm}^{2}\right)$ & $85 \pm 8$ & $65 \pm 7$ \\
\hline \hline
\end{tabular}

* As marked by the greem bars in Figure $\square$

** Total fluence of the line in the respective flares

The fluxes of the X1 flare obtained with SPI and GBM differ by $\sim 30 \%$ at $2.2 \mathrm{MeV}$, which might result from the complex nature of GBM background subtraction, as well as a systematic difference between SPI and GMB due to e.g. possible inaccuracies of the mass model of INTEGRAL used in the Monte-Carlo simulations.

\section{Acknowledgements}

The authors would like to acknowledge helpful discussions with Dr. Gerald Share. ZXL is grateful for DLR support (contract No. FKZ 50 OG 0502).

\section{References}

[1] Ramaty, R., Kozlovsky, B., \& Lingenfelter, R. E. 1979, ApJS, 40, 487

[2] Share, G. H., Murphy, R. J., Skibo, J G.. et a;/ 2003, ApJ, 595, L85

[3] Gros, M., Tatischeff, V., Kiener, J., et al. 2004, 5th INTEGRAL Workshop on the INTEGRAL Universe, 552, 669

[4] Share, G H., Murphy, R. J., Smith, D. M., et al. 2004, ApJ, 615, L169

[5] Kiener, J., Gros, M., Tatischeff, V., \& Weidenspointner, G. 2006, AAp, 445, 725

[6] Gruber, David, in preparation

[7] Meegan, C., Lichti, G., Bhat, P. N. et al. 2009, ApJ, 702, 781

[8] Fitzpatrick, G., Connaughton, V., McBreen, S. \& Tierney, D. 2011, arXiv:1111.3779 